\title{
Emotion Detection Using Convolution Neural Network, Expert System and Deep Learning Approach
}

\author{
Prabha Seetaram Naik', Dipti Patnayak ${ }^{2}$ and S.Geetha*3 \\ ${ }^{1}$ Department of Computer Science, Nagarjuna College of Engineering and Technology, Bangalore, India \\ ${ }^{2}$ Department of Computer Science, M. S. Engineering College, Bangalore, India \\ ${ }^{3}$ Department of Information Science and Engineering, CMR Institute of Technology,Bangalore, India
}

\section{ABSTRACT}

This work presents a facial expression identification system using the Facial Action Coding System with the use of the Bezier curves approximation method. This technique uses the features of the human face. These extracted face expressions are done with the idea of the face geometry and are also approximated by 3rd order Bezier curves by illustrating the relationship between the face feature movement and by observing the change of expressions. For face feature identification, color segmentation is done with the help of fuzzy logic classification which minimizes color similarities. Result outcomes define that this technique can identify the facial expressions with an accuracy of more than ninety cases. From human face structure, we divide into four regions such as right eye, left eye, nose, and mouth areas from the face image. Firstly, comes the face detection and the detection of the skin region. We crop the facial skin region and connect the largest skin region to detect the skin surface of the human face. When the emotion is perceived, the system recommends a play-list for the images. Based on the facial emotions, the Musical recommendation system creates a list of suggestions for music that are ranked from top to bottom.

KEY WORDS: FACIAL ACTION CODING SYSTEM (FACS) - BEZIER CURVES, FACE FEATURES IDENTIFICATION, FACE EMOTION DETECTION, CNN, DEEP LEARNING, MUSICAL RECOMMENDATION SYSTEM.

\section{INTRODUCTION}

In the wide range of applications in the field of human machine interaction, face feature extraction method has played considerably important role and has been attracting plenty attention as it provides easy and natural way to interact with humans (Babu et.al, 2020). Few of the application areas which shows prominent improvement in human machine interaction include personal identification and access

\section{ARTICLE INFORMATION}

*Corresponding Author: geetha2016reserach@gmail.com Received 16th Oct 2020 Accepted after revision 31st Dec 2020 Print ISSN: 0974-6455 Online ISSN: 2321-4007 CODEN: BBRCBA

Thomson Reuters ISI Web of Science Clarivate Analytics USA and Crossref Indexed Journal

\section{Clarivate
Analytics}

NAAS Journal Score 2020 (4.31)

A Society of Science and Nature Publication,

Bhopal India 2020. All rights reserved.

Online Contents Available at: http//www.bbrc.in/

Doi: http://dx.doi.org/10.21786/bbrc/13.13/34 control, video phone and teleconferencing, forensic applications, automated surveillance, cosmetology, and many more. There is a developing requirement for face acknowledgment frameworks that are quick, exact regardless of the environment (Jaiswal and Nandi 2020). Face acknowledgment is an undertaking of example acknowledgment that is explicitly performed on countenances. It arranges a face either as known or obscure by contrasting it with put away faces in the information base. There are three significant ways to deal with face acknowledgment calculations, to be specific frontal, profile, and view open minded acknowledgment.

All face acknowledgment calculations have three phases: Preprocessing, Feature Extraction, Training and Arrangement. Preprocessing includes extricating face districts and separating them for additional handling. Picture separating employments picture denoising strategies that are frequently founded on the

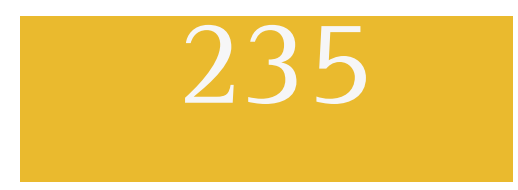


minimization of energy capacities. Absolute variety denoising (Television denoising) is one such strategy that jam significant data, for example, edge sharpness and area, however may bargain on some picture highlights, for example, surface (Geetha et.al,2020). Boisterous and insignificant information are significant deterrents to characterization, hence preparing the model to choose significant highlights is a basic advance.

The objective is to choose a subset of significant measurements while eliminating the superfluous ones (Joseph and Geetha,2020). These measurements don't change essentially and can adjust to new classes and keep a high connection among the components inside them, even without retraining. There are numerous acknowledgment calculations, for example, character, voice and essential example acknowledgment that can run on low end gadgets with limited preparing force and memory. Yet, not many face acknowledgment calculations can run proficiently on those low end gadgets (Pranav,2020). There are two primary methodologies for face acknowledgment, utilization of numerical models (either layout or calculation based) or AI. Various kinds of neural organizations, for example, Profound, Convolutional or Hybrid (Alreshidi and Ullah, 2020) can perform face acknowledgment for various stances and enlightenments adequately, in any case, require a great deal memory to store the prepared model. They moreover require impressive preparing capacity to run face acknowledgment.

This makes them unsatisfactory for utilization in low end gadgets except if the gadgets are explicitly improved for them. (Geetha and Deepalakshmi,2020)Numerical models for face acknowledgments use capacities for example, LBP, Eigen, DCT, SIFT, PCA, Gabor and Markov models. There are numerous that can perceive faces at various enlightenments and postures (Giuseppe et.al,2019). These can run on explicit gadgets or on FPGAs after advancements. One of the issues with Mathematical model is the need to retrain the framework for each new face that is added. The preparation framework will in general be more memory and cycle concentrated, and retraining the framework for each face turns into a weight.

An opportunity to prepare is influenced by changes in the client's setting as individuals are added and taken out from the framework (Homsapaya et.al,„2019). The explanation for picking Pearson relationship rather than capacities, for example, Mahalanobis distance (Geetha and Deepalakshmi,, 2019) is to take out the requirement for retraining on account of changes in the covariance grid. This calculation has been assessed 2021 International Conference on Computing, Communication, and utilizing standard face datasets like ORL (Brandon et.al,2016), MUCT Labeled Faces in the wild (LFW) (Biswas et.al,2016). LFW Dataset is considered the most mind-boggling dataset as it incorporates a ton of posture, lighting and quality varieties. In this paper, a facial expression identification system is implemented with the use of the Facial Action Coding System.
Figure 1: Face Identification Procedures

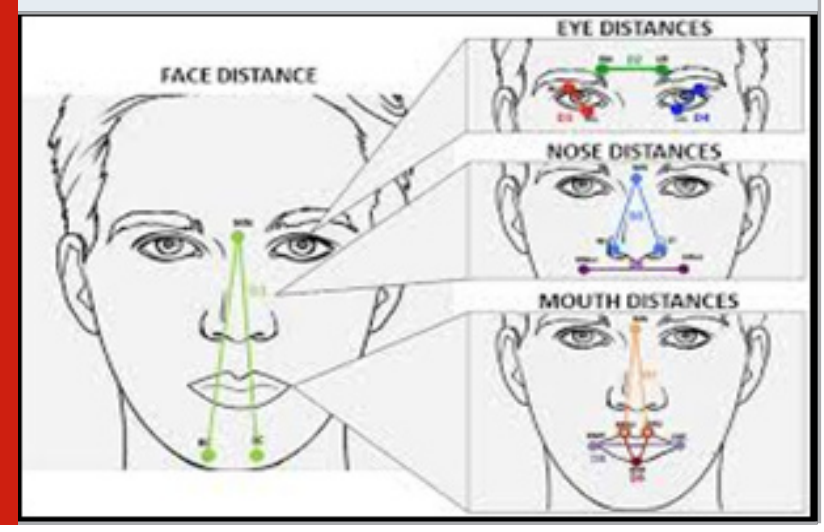

A number of face identification procedures and algorithms are used in today's technology. Some classification is holistic in nature and few are human interaction that is knowledge based. Figure 1 refers the face features for extraction. Some are also based on the data extracted from the human face. Each of this classification has merits and lacunas. When these techniques are applied individually, they might not be able to address most of the problems including expression, pose or orientation. Hence, always recommended to use multiple techniques in succession. Six primary face expression identification techniques are smiling face of a person, face filled with sad moments, scared face, aggression, revulsion face and intense sorrow face. Motion of the muscles of the face are grouped into forty-four action units and any facial expression are illustrated by these six primary combinations(Geetha and Deepalakshmi,, 2019).

Related Works: The process of identifying people through facial images, has numerous practical applications in the area of biometrics, information security, access control, law enforcement, smart cards and surveillance system. Convolutional Neural Networks(CovNets), a type of deep networks has been proved to be successful for FR. For real- time systems, some preprocessing steps like sampling needs to be done before using to CovNets. But then also complete images (all the pixel values) are passed as input to CovNets and all the steps (feature selection, feature extraction, training) are performed by the network(Antonin and Thomas , 2016)).

This is the reason that implementing CovNets are sometimes complex and time consuming. the process of identifying people through facial images, has numerous practical applications in the area of biometrics, information security, access control, law enforcement, smart cards and surveillance system. Convolutional Neural Networks(CovNets), a type of deep networks has been proved to be successful for FR. For real- time systems, some preprocessing steps like sampling needs to be done before using to CovNets. But then also complete images (all the pixel values) are passed as input to CovNets and all the steps (feature selection, feature extraction, training) are performed by the network. This is the reason that implementing CovNets are sometimes complex and time consuming(Geetha et.al,2020). 
Eye blink recognition, and face recognition are very popular and promising techniques. In some cases, people can use the photos and face masks to hack mobile security systems,so we propose an eye blinking detection,which finds eyes through the proportion of human face. The proposed method detects the movements of eyeball and the number of eye blinking to improve face recognition for screen unlock on the mobile devices.A paper on Automatic face recognition is now widely used in applications ranging from de duplication of identity to authentication of mobile payment. This popularity of face recognition has raised concerns about face spoof attacks (also known as biometric sensor presentation attacks), where a photo or video of an authorized person's face could be used to gain access to facilities or services (Laurent, 2013).

A review of Face Recognition Based Car Ignition and Security System, the face recognition-based car ignition system literally replaces the car ignition by replacing the key with specific user face (Geetha, and Deepalakshmi,2019). While dealing with the topic the objective arises is the achievement of luxurious features and the safety concern, which can be achieved by means of the automotive electronics(Guo et.al,2016). In this paper, we are proposing facial recognition system by embedding face detection and face tracking system algorithm found in a method using thermal image processing and a neural network is presented for recognition of facial expression. The method is based on 2-dimensional detection of temperature distribution of the face, using infrared rays. The front-view face in the input image is normalized in terms of the size and the location, followed by measurement of the local temperature difference between the averaged neutral and the unknown expression faces.

Figure 2: Preparing the face picture to extricate

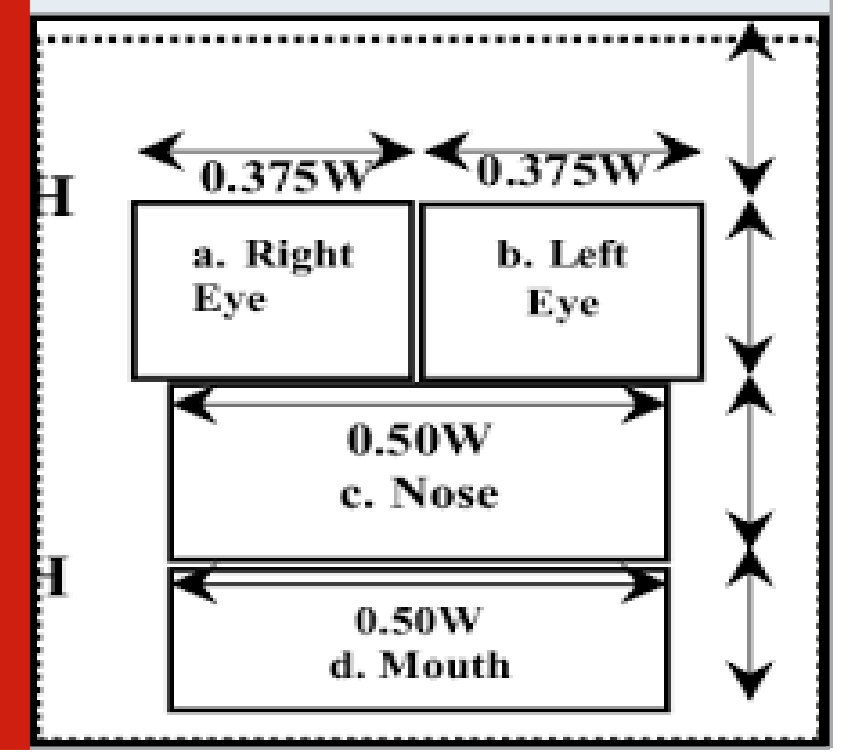

Proposed System: In the proposed system we overcome the problems that we face in the existing system as we are going for more conventional and an accurate way of dealing with a person's behavior Here, we mainly use person's facial expressions to decide whether he or she is happy, sad, neutral, angry, surprised or any other behavior a person depicts to identify and find the suitable posts that favors his progressing behavioral aspects. For this process(Fig 2), Facial Action Coding System extraction procedure we use; A depth edge detection by image based smoothing.Basic cross sectional segment to confine extraction,Facial landmark localization technique near Nose and mouth region.

Figure 2 refers the extricate measurements for face feature extraction. The face acknowledgment procedures depend on preparing the face picture to extricate important highlights and train the classifier to foresee the class of the picture. As examined over, the cycle includes the accompanying advances:

1. Preprocess the picture

2. Extract highlight vector

3. Train the model - train the classifier

4. Test the model - Enrollment and Recognition prepared model to foresee the class of the picture for the emotion

5. Playing the Musical Songs will be prioritized.

Figure 3: Steps to Extract Facial Features Output

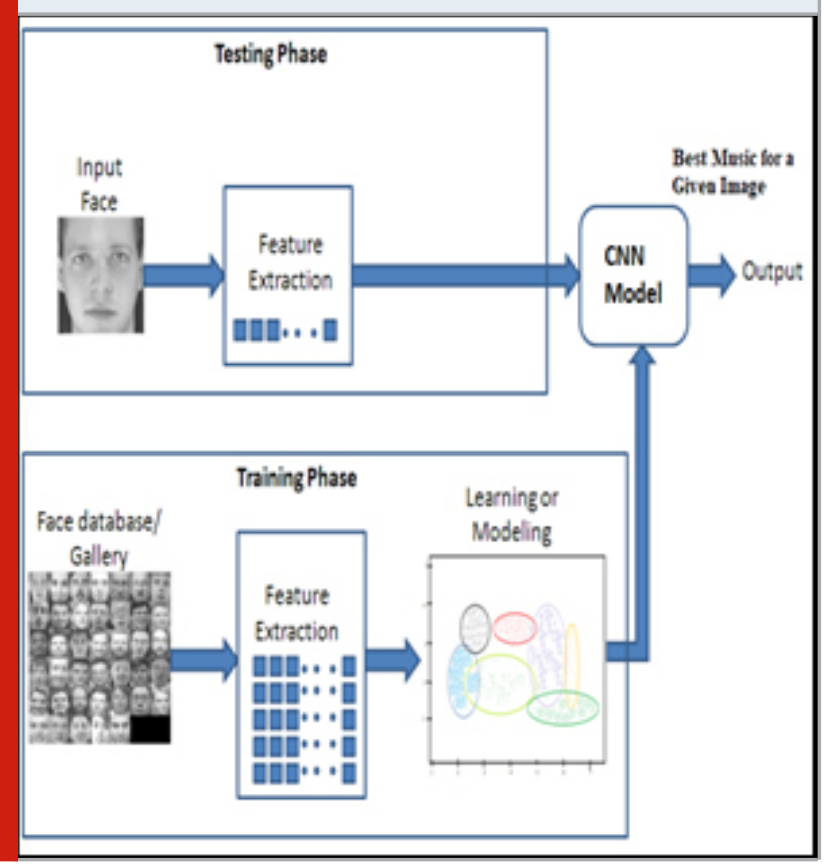

Existing systems are very complex in terms of time and memory requirements for extracting facial features in real time. Based on the current emotional state and behavior of a user, existing systems possess a lesser accuracy in generation of a playlist. Some existing systems tend to employ the use of human speech or sometimes even the use of additional hardware for generation of an automated playlist, thereby increasing the total cost incurred. Some existing systems tend to employ the use of human speech or sometimes even the use of additional hardware. Figure 3 refers the complete 
scenario of actions and how the final result is computed from various modules.

\section{METHODOLOGY}

There is some overlap with disciplines of systems analysis, systems architecture and systems engineering. The input which is the face is taken from the web camera, this input will be recognized and sent to the processing stage where the face detection is done. Once the processing of the given input is done it undergoes the identification of the emotion in the input and based on the identified emotion required song will be played. The machine can be trained with models which will help in identifying the emotion from input by using LBP (Local Binary Patterns) extraction and PCA (Principal Component Analysis) so that the emotions. Figure 4 shows the flowchart with image classification.

Figure 4: Flow chart for Classifying the image with CNN

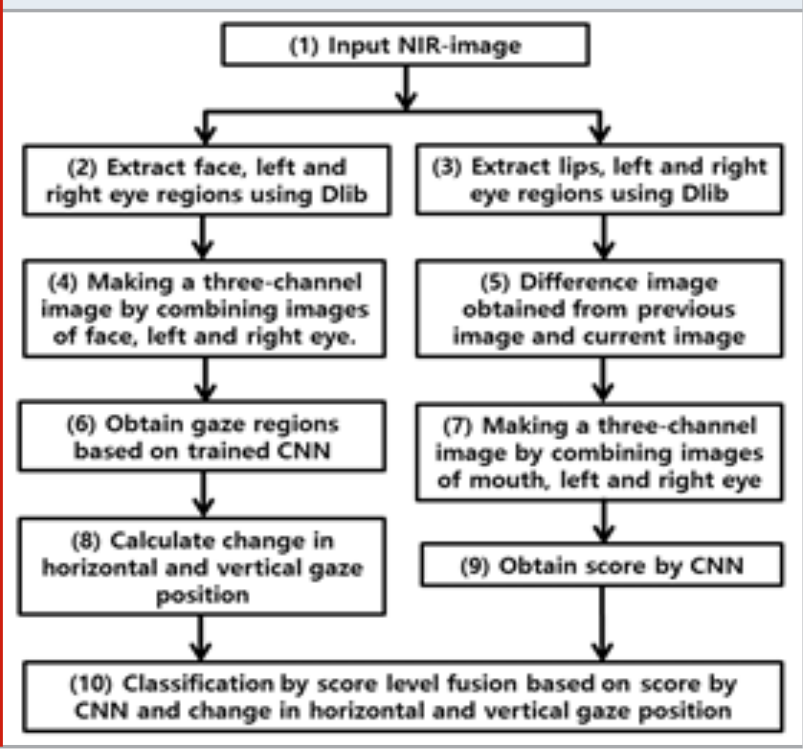

Facial Feature Extraction Module: This model uses Convolution Neural network (CNN), to identify the human emotion, the protocol uses data which has many images and these images depicts basic human facial expressions like smiling face of a person, face filled with sad moments, scared face, aggression, revulsion face and intense sorrow face. Here the system is trained using these types of images. CNN has systematic selflearning approach.

The initial step is to resize the face picture to a fixed measurement and denoise the picture as appeared in Fig. 1 imperative to safeguard sharp discontinuities to keep exact recognizable proof of picture edges. Complete variety denoising (Television denoising) is strikingly successful at all the while saving edges while smoothing endlessly commotion in level locales, indeed, even at low sign to-clamor proportions. In this paper, we propose utilizing the over CNN calculation (Algorithm 1) to denoise the face picture. The functions used in the algorithm are given in (1) to (5). $(x)=\operatorname{cat}(3,[\operatorname{diff}(x, 1,1)],[\operatorname{diff}(x, 1,2]$ $\operatorname{zeros}(\square, 1)]) \operatorname{zeros}(1, w)$

$$
\begin{aligned}
& D^{\sim}(x)=[x(1,:, 1) \operatorname{diff}(x(:,::, 1), 1,1) M(x)]- \\
& {[x(:, 1,2) \operatorname{diff}(x(:,:, 2), 1,2)]}
\end{aligned}
$$

$$
\begin{aligned}
& M(x)=\sqrt{\left(\left(€\left(x^{2}\right), 3\right) / \gamma\right)} \\
& \operatorname{proxcF}(x)=(\mathrm{x}+(\mathrm{c} * \mathrm{iNg}))(1+\mathrm{c}) \\
& \operatorname{proxcG}(x)=\mathrm{x}(\max (\mathrm{M}(\mathrm{x}), 1))
\end{aligned}
$$

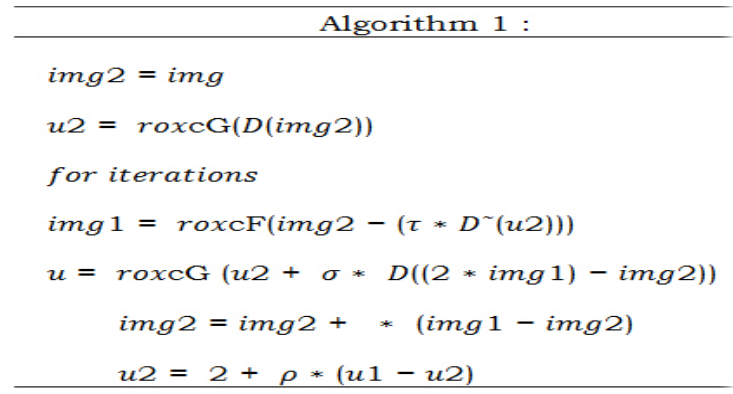

With each and every connection there are associated weights present. This increases the possible computational complexities that are much likely to occur. But then in CNN, all the nodes that is present in a particular layer are being connected to only valid nodes in the succeeding layer. Thus there will be minimal computational complexities. This then includes various layers, in which training and testing input images are to be done. The final layer is wholly connected which performs classification tasks which in turn classifies images in accordance to emotions.

Figure 5: Same-id set mapping

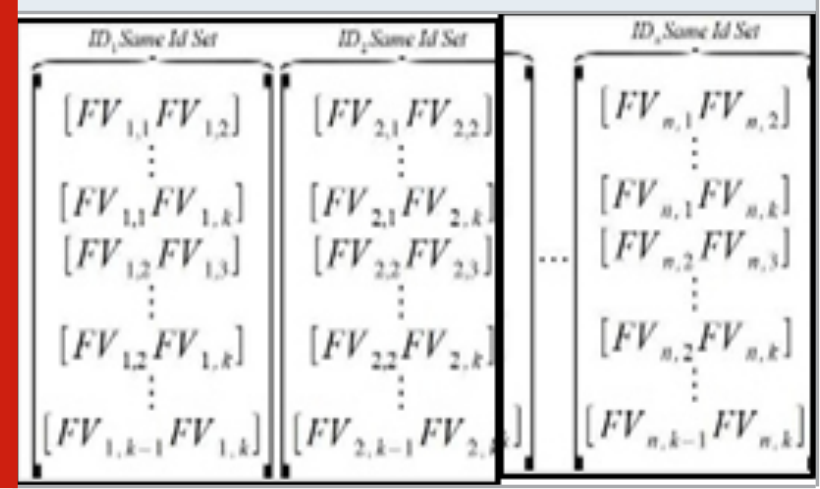

Emotion Detection and Song Classification Module: This is then represented as a three-dimensional matrix and various operations are or is to be done on this particular matrix, mostly for training and testing. As a further matter, in some of the other neural networks like for an example in fully connected networks, Each and every node in a particular layer is connected to each and every 
node in the succeeding layer. Algorithm 1 executed and resulted with the matrix of Figure 5.

Change based methodology utilizing moving window 2D-FFT is utilized for FV extraction. In this methodology, every pixel is important for 3 sub-windows. The primary 2D-FFT is performed on the whole picture, the second arrangement of 2D-FFTs is performed on windows $1 / 2$ the size of the picture and moving a distance $1 /$ fourth the size of the picture to the following window. The last arrangement of 2D-FFTs is performed on windows 1/ fourth the size of the picture and moving a distance of 1/eighth the size of the picture. Outright estimations of 2D-FFT for every window is straightened and annexed to the FV cluster.

It isn't practical to utilize the whole dataset for classifying the data sets as it can turn out to be computationally comprehensive. Just a preset number of information focuses in same and cross-id datasets are needed for every ID to make the image preparation productive and computationally suitable. The means are as per the following:

\section{Algorithm 2}

1. In the equivalent id datasets of image points, select the FV mixes that cover the whole relationship opportunities for every ID. To get this, sort the relationship esteems and pick required number of information focuses at customary spans over the whole scope of arranged qualities.

2. In cross-id datasets of image, utilize a similar cycle as above.

To try not to any train inclination, guarantee to have a similar number of information focuses in same and cross-id datasets. Algorithm 2 executed and resulted with the matrix of Figure 6.

The matrix contains records and every double component decides whether that specific list is to be considered in connection or not. Process the relationship for each set in the equivalent and cross-id datasets for all the records. The wellness work figures out which records bring about expanding the hole between the equivalent and cross-id relationships.

$$
\begin{gathered}
C= \\
\frac{\sum_{1}^{n}\left(x_{i}-\bar{x}\right)\left(y_{i}-\bar{y}\right)}{\left(\sqrt{\left(\sum_{1}^{n}\left(x_{i}-\bar{x}\right)^{2}\right)\left(\sum_{1}^{n}\left(y_{i}-\bar{y}\right)^{2}\right)}\right) \sum(x i-x) 2 *(y i-y) 2}
\end{gathered}
$$

In the above equation $\mathrm{x}$ and $\mathrm{y}$ are the Feature vectors that need to be correlated, $\mathrm{x}^{-} \mathrm{y}^{-}$and $\mathrm{y}^{-}$represent the mean of the feature vectors respectively and $n$ represents the total number of elements in the feature vectors. Start with a random population set of points and the individuals with the best fitness mate to create the next set. The individual with the best fitness score, after the final iteration, represents the final selection of the output of Neural Network with four expressions: smile, aggression, sorrow and neutral. The image with the best wellness score, after the last emphasis, speaks to the last choice of selection of song. The fitness function is defined (7) such that the individual that gives the lowest value creates the biggest gap between same and cross-id correlations and is the best solution

\section{Fitness_score $=1-((1-c) *(1-$ $\left.\left.\sigma_{\mathrm{c}}\right)\right)(\mathrm{x}-c)$

In the above equation, $\sigma_{\mathrm{c}}$ and $\mathrm{x}$ represent the standard image is expected to have a high correlation closer to and cross-id dataset is expected to have a low correlation closer to 0.0. It is to be noted that the cross-id image points will have many more combinations compared to the same-id combinations. The wellness work is characterized (7) with the end goal that the image that gives the most minimal worth makes the greatest hole among same and cross-id relationships and is the best selection of songs. The system is designed in such a way that when the emotion is figured out, list of songs related to that particular expression will be shown up on the screen. Songs will be prioritized and selected then played.

Experimental Result: Testing and implementation is done successfully on the windows 10, 64-bit operating system with i5 core processor. The face recognition is done using the Deep Neural Network technique and Convolution Neural Network.

CNN are the neural network architecture which has multiple layers. Input and output of CNN are array vectors known as feature map. TABLE I. refers the size of the image used in each module. The type of input defines the array dimensions. An example would be, the audio input has one dimensional array as well as text input; the image has a $2 \mathrm{D}$ array. The features that are extracted from input well describes the output feature map. CNN comprises of mainly three layers: convolutional filter layer, pooling/subsampling layer, and classification layer. The paramount limitations of feature-based approaches are that big efforts should be put on to design and employ various feature extraction methods which are human crafted features. As for overcoming this drawback, we are proposing a new approach that is purely based on deep learning, a machine crafted feature that does the automatic extraction of the facial features. Identify the best music for a given image is performed by fitness score that was tested by the following parameters.

As for the music recommendation system developed Collaborative filtering algorithm technique is being used, Collaborative filtering is a known technique that can be 
used to filter out items that a particular user might or does like on the basis of reactions by other similar users. It toils by finding a large group of people and searching for a smaller set of users with likes that are similar to a particular user himself.

It looks up at the plethora of items that they fancy and are then combined to create a list of suggestions that are ranked from top to bottom is displayed with Table II. When Algorithm 2 executed and resulted with the matrix and it was interpreted with the result of surprise emotion for image classification with 32 persons. For executing Algorithm face emotions of 32 person was considered with the distance relative to face diagonal.

When Algorithm 2 executed and resulted with the matrix and it was interpreted with the result of emotions classification with 32 person.
Figure 6: Cross-Id Datasets Mapping

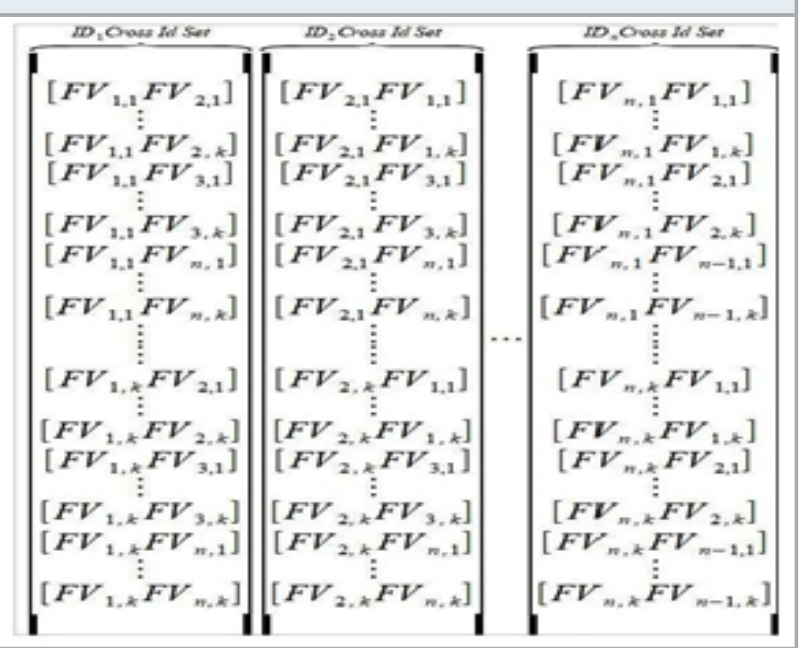

\begin{tabular}{|l|c|c|c|}
\multicolumn{4}{|c|}{ Table 1. Requirements Consideration at Image Size In Differ Modules } \\
\hline S.No & $\begin{array}{c}\text { Face Resize } \\
\text { Size }\end{array}$ & $\begin{array}{c}\text { of the image observed } \\
\text { for feature } \\
\text { extraction }\end{array}$ & $\begin{array}{c}\text { Size of the image } \\
\text { observed for } \\
\text { classification of Emotion }\end{array}$ \\
\hline 1. & $16 \times 16$ & $10 \mathrm{~kb} \sim 2 \mathrm{kB}$ & $\sim 800 \mathrm{kB}$ \\
\hline 2. & $32 \times 32$ & $40 \mathrm{~kb} \sim 8 \mathrm{kB}$ & $\sim 3200 \mathrm{kB}$ \\
\hline 3. & $128 \times 128$ (spatial) & $640 \mathrm{~kb}$ & $640 \mathrm{~kb}$ to $256000 \mathrm{~kb}$ \\
\hline
\end{tabular}

Table 2. Number of Iterations for Identifying Music Ranking

\begin{tabular}{|l|c|c|}
\hline S.No & Face Resize & $\begin{array}{c}\text { Preprocessed Image } \\
\text { Iterations for Identifying } \\
\text { Music Ranking }\end{array}$ \\
\hline 1 & $16^{*} 16$ & 18 \\
\hline 2 & $32 * 32$ & 32 \\
\hline 3 & $64^{*} 64$ & 37 \\
\hline 4 & $128^{*} 128$ & 39 \\
\hline
\end{tabular}

Figure 7: Emotion Detection with Face Features

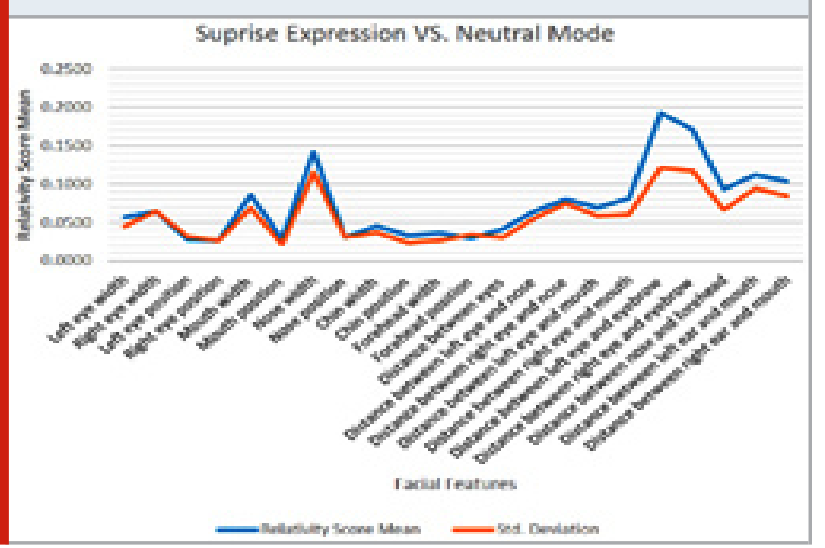

Figure 8: Comparison Chart for Face Features With 35 Person With 2 Frames

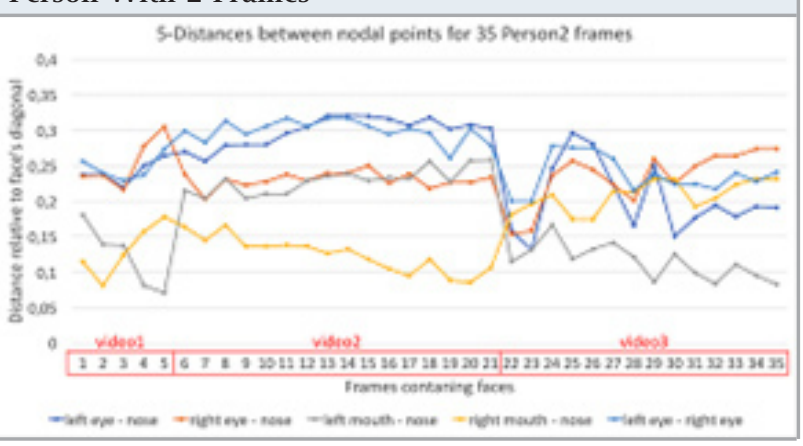

\section{CONCLUSION}

In our paper the facial expression recognition is implemented to classify human facial expression i.e., Happy, Sad, Surprise, Anger and Neutral. The system has been evaluated using accuracy and precision, based on this the user will be presented with certain music. When the emotion is perceived, the framework recommends a play-list for that feeling, saving of time for a client over choosing and playing melodies physically. Feeling Based Music Player additionally monitors client's subtleties like number of plays for every melody, sorts tune dependent 
on class and interest level, and revamps the play-list without fail. The framework additionally informs client about the melodies that are never played so they can be erased or changed will be implemented in future and the speed of recognition in nano seconds will be implemented.

\section{REFERENCES}

Babu, R. G., Maheswari, K. U., Zarro, C., Parameshachari, B. D., \&t Ullo, S. L. (2020). Land-Use and Land-Cover Classification Using a Human Group-Based Particle Swarm Optimization Algorithm with an LSTM Classifier on Hybrid Pre-Processing Remote-Sensing Images. Remote Sensing, 12(24), 4135.

Jaiswal, S. and Nandi, G.C., (2020). Robust real-time emotion detection system using cnn architecture. Neural Computing and Applications, 32(15), pp.11253-11262. Geetha, S., Nanda, P., Raj, R.J.S. and Prince, T., Early Recognition of Herb Sickness Using SVM. In Intelligence in Big Data Technologies-Beyond the Hype (pp. 543550). Springer, Singapore.

Joseph, A. and Geetha, P., (2020). Facial emotion detection using modified eyemap-mouthmap algorithm on an enhanced image and classification with tensorflow. The Visual Computer, 36(3), pp.529-539.

Pranav, E., Kamal, S., Chandran, C.S. and Supriya, M.H., (2020), March. Facial emotion recognition using deep convolutional neural network. In 2020 6th International Conference on Advanced Computing and Communication Systems (ICACCS) (pp. 317-320). IEEE.

Alreshidi, A. and Ullah, M., (2020), March. Facial Emotion Recognition Using Hybrid Features. In Informatics (Vol. 7, No. 1, p. 6). Multidisciplinary Digital Publishing Institute.

Geetha, S. and Deepalakshmi, P.,( 2020). Rapid retrieval of secured data from the sensor cloud using a relative record index and energy management of sensors. International Journal of Intelligent Enterprise, 7(1-3), pp.3-14.

Giuseppe Schirripa Spagnolo, Lorenzo Cozzella, Fabio Leccese,( 2019) Phase correlation functions: FFT vs. FHT, IMEKO ISSN: 2221-870X March, Volume 8, Number 1, 87-92

Homsapaya, Kanyanut \&t Sornil, Ohm. (2018). Modified Floating Search Feature Selection Based on Genetic
Algorithm. MATEC Web of Conferences. 164. 01023. 10.1051/matecconf/201816401023.

Geetha, S. and Deepalakshmi, P., (2019). Enhanced Energy in Sensors by Avoiding Voids and Saving Sensitive Data on Cloud Using B+ Tree Index with Retrieval of Query Predicates. Mobile Networks and Applications, 24(1), pp.234-247 016/j.ijleo.2015.08.221.

Brandon Amos, Bartosz Ludwiczuk, Mahadev Satyanarayanan, (2016)OpenFace: A general-purpose face recognition library with mobile applications, CMUCS-16-118

S. Biswas, G. Aggarwal, P. J. Flynn and K. W. Bowyer, (2013)Pose-Robust Recognition of Low-Resolution Face Images, in IEEE Transactions on Pattern Analysis and Machine Intelligence, vol. 35, no. 12, pp. 3037-3049, doi: 10.1109/TPAMI.2013.68.

Geetha S, Deepalakshmi P.(2020) Rapid retrieval of secured data from the sensor cloud using a relative record index and energy management of sensors. International Journal of Intelligent Enterprise.;7(1-3):314.

Antonin Chambolle, Thomas Pock, (2016)An introduction to continuous optimization for imaging, Acta Numerica, Cambridge University Press (CUP), Acta Numerica, 25, pp.161-319. 10.1017/S096249291600009X. Hal01346507

Geetha, S., Deepalakshmi, P. and Pande, S., (2019), December. Managing Crop for Indian Farming Using IOT. In 2019 IEEE International Conference on Clean Energy and Energy Efficient Electronics Circuit for Sustainable Development (INCCES) (pp. 1-5). IEEE. Laurent Condat, (2013)A primal-dual splitting method for convex optimization involving Lipschitzian, proximable and linear composite terms, Journal of Optimization Theory and Applications, Springer Verlag, ff10.1007/s 10957-012-0245-9ff. ffhal-00609728v4f Geetha, S. and Deepalakshmi, P., (2019), April. Proactive Mechanism of Replacing Void Sensor and Managing Cluster heads. In 2019 IEEE International Conference on Intelligent Techniques in Control, Optimization and Signal Processing (INCOS) (pp. 1-6). IEEE.

Guo, Li \&t Chen, Weilong \&t Liao, Yu \& Liao, Honghua \& Li,(2016) A edgepreserved image denoising algorithm based on local adaptive regularization, Optik International Journal for Light and ElectronOptics. 10.1 